
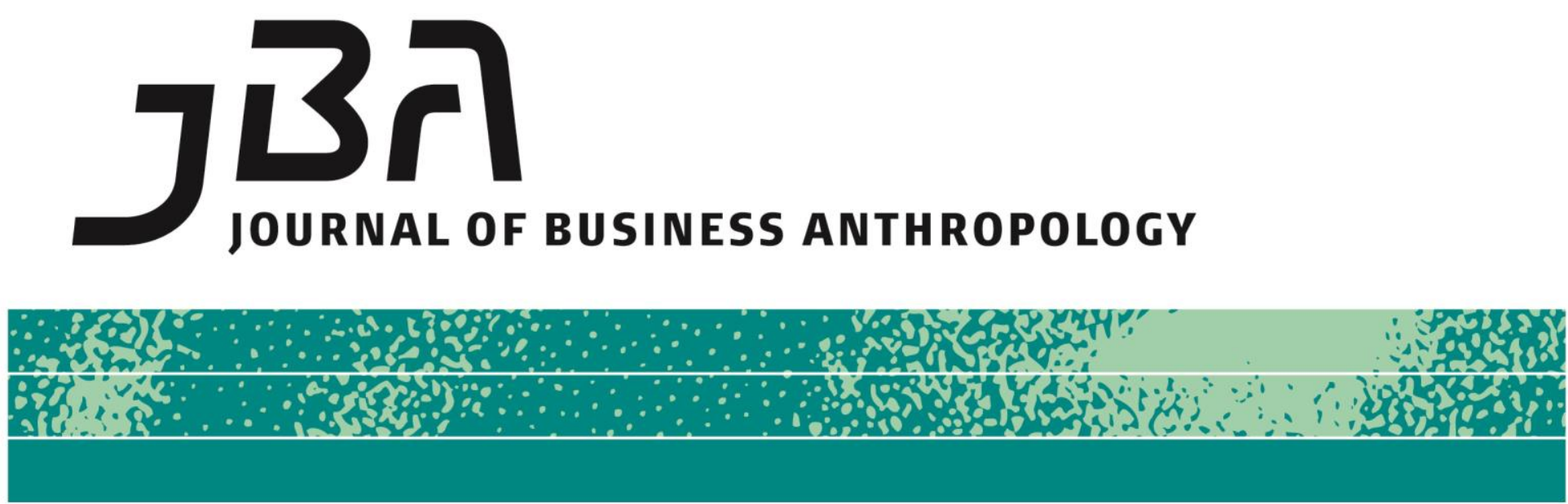

\title{
Towards Transdisciplinarity: Liminality and the Transitions Inherent in Pluridisciplinary Collaborative Work
}

\author{
Christine Z. Miller
}

\begin{abstract}
Although the value of cross-disciplinary teams is widely accepted, relatively little attention has been given to the work that precedes addressing a team's objective or stated problem, that is, the work required to negotiate their various disciplinary perspectives. This article considers how the notion of liminality, a cultural and social state of "betweenness," might be used to conceptualize transitory stages in the development of pluridisciplinary groups and teams that are comprised of individuals from many diverse disciplines. It suggests how anthropologists can play a role in guiding and facilitating this particular domain of invisible work.
\end{abstract}

\section{Keywords}

Liminality, invisible work, pluridisciplinary, interdisciplinary, multidisciplinary, transdisciplinary teamwork 
"Life is multiple disciplinary. Disciplines are the result of the artificial fragmentation of knowledge."

(Choi and Pak 2006:357)

\section{Introduction}

The increasing use of cross-disciplinary teams in business and academic settings reflects a growing awareness of the limitations of singular forms of inquiry to address the complex problems in societies and organizations (Conklin 2005, Johnson 2009). All too often, the dominant approach to problem solving has involved parsing out bits and pieces to individuals or homogeneous teams and then attempting to bundle the results together. However, a paradigm shift is currently underway that acknowledges different classes of problems, some of which require a multilevel approach and the integrated perspective of multiple disciplines. The atomistic or reductionist approach to problem solving that is characteristic of single disciplinary problem solving approaches typically favors one perspective over others and often generates unintended consequences that create more problems than are solved. For example, urban renewal projects that vaporize existing "blighted" neighborhoods and relocate residences to multistory mega housing blocks have failed to produce the desired results. Many now stand vacant, haunting testaments to single-source solutions to complex multidimensional problems involving social, cultural, economic, technological, and environmental components. An emerging sense of the complexity of many of the problems faced by contemporary societies has engendered a search for alternatives that incorporate multiple points of view and disciplinary perspectives.

Choi and Pak (2006:351) suggest that the phrase "multiple disciplinary" be used as a general term "for when the nature of involvement of multiple disciplines is unknown or unspecified." In this article, the term pluridisciplinary is substituted for multiple disciplinary to avoid confusing multiple disciplinary and multidisciplinary, which represent two distinct forms of team members' engagement and interaction. Pluridisciplinary serves not only as a general term, but also as a rubric under which three forms or stages of teaming-multi-, inter- and transdisciplinary-are encompassed. As the deployment of pluridisciplinary teams is increasing, the unique challenges of this work have begun to surface. If these teams are to deliver innovation and framechanging solutions that can be achieved through requisite variety and heterogeneity, then they must overcome the barriers embedded in singular disciplinary perspectives. This work is inherently uncomfortable as it requires openness to accepting diverse knowledge domains and ways of working that may conflict with one's own assumptions, disciplinary orientation and work practices. 
If team members are unable or unwilling to embark on what can be a significant learning curve, then barriers to effective teaming within cross-disciplinary groups can derail projects and goals. Because people bring their cultural (here in the sense of disciplinary) differences in language, tools, work practices, epistemologies, paradigms, and goals relative to the project, there is a need to better understand the processes involved in team formation and establishing a shared understanding of the mission and objectives. There is work to be done before, during, and after the teaming experience to assist the team in navigating the transitions from a group representing single disciplines to a unified team that transcends individual disciplinary boundaries and perspectives. The purpose of this article is to consider how liminality as a cultural and social state of in-between-ness and a phase in the ritual process might be used to conceptualize transitory stages in the development of pluridisciplinary groups and teams.

The role of the ritual guide is suggested as an analogy for individuals who play a key role in assisting teams to navigate the stages of cross-disciplinary teamwork. The literature on group and team formation supports the claim that groups and teams go through various stages in their development. Tuckman's classic article on small group development has become the basis for much subsequent research on phases of group and team development. He identified four stages in group development that could be distinguished by those that describe the social or interpersonal activities among group members and those that describe the task work of the group. "In the social realm, these stages in the developmental sequence are testing-dependence, conflict, cohesion, and functional roles. In the task realm, they are orientation, emotionality, relevant opinion exchange, and the emergence of solutions (Tuckman 1965:384). The four stages were later abbreviated to "forming," "storming," "norming," and “"performing" (Tuckman and Jensen 1977).

Early studies of the stages of group development could hardly have anticipated twenty-first century conditions, particularly the impact of advances in technology, and the deployment of pluridisciplinary teams facing the unique challenges of cross-disciplinary collaboration. Differences in language, socialization, tools, and work processes can pose formidable barriers and often limit-if not completely derail-the work of teams composed of members from multiple disciplines. By applying the lens of liminality as a "mediating period" (Barfield 1977) that defines a transition in a rite of passage, this article considers the interactions that occur in the transitions between the stages of interpersonal group development. It also suggests how Forsythe's (1999) concept of "invisible work" might be applied to the processes entailed in moving from one stage to another. Further, the article discusses how anthropologists in particular can guide and facilitate the transitions between multi-, inter-, and transdisciplinarity. 


\section{Differentiating between multidisciplinarity, interdisciplinarity, and transdisciplinarity}

Applying the concept of liminality to explore the differences between multidisciplinary (additive), interdisciplinary (integrative), and transdisciplinary (holistic) teams allows for examining these types of teams as varying points on a continuum of cross-disciplinary work (Choi and Pak 2006). Although it is common to see the terms used interchangeably, there are important qualitative differences among these modes of interaction in pluridisciplinary work (Margolin 2002, Conklin 2006, Choi and Pak 2006, Strathern 2007, Lawrence 2010, McGregor 2014) Differentiating between the terms allows for important distinctions between the stages through which teams might progress. Progression through multidisciplinarity, interdisciplinarity, and transdisciplinarity is neither guaranteed nor compulsory. Not all situations require the attention of a pluridisciplinary team. As Choi and Pak note "While multiple disciplinary [i.e., pluridisciplinary] teamwork is appropriate for complex problems, it is not always necessary in every single project."

Recognizing that multi-, inter-, and transdisciplinary tend to be used interchangeably, Choi and Pak (2006:351) conducted a study of how the three terms were applied in academic literature, ultimately consolidating their findings to provide these definitions:

Multidisciplinarity draws on knowledge from different disciplines but stays within their boundaries. Interdisciplinarity analyzes, synthesizes and harmonizes links between disciplines into a coordinated and coherent whole. Transdisciplinarity integrates the natural, social and health sciences in a humanities context, and transcends their traditional boundaries.

Strathern (2007) echoes Choi and Pak in acknowledging the crucial distinction among the three terms. Referring to Nowotny's contribution to discussions regarding "the potential of transdisciplinarity" (Gibbons et al. 1994, Nowotny, Scott, and Gibbons 2001), Strathern (2007:124) notes:

Interdisciplinarity, in the strict sense, points to a framework shared across disciplines to which each contributes a bit. (Multidisciplinarity, the simple alignment of skills from different disciplines, is already left behind.) Transdisciplinarity implies even more: it brings disciplines together in contexts where new approaches arise out of the interaction between them, but to a heightened degree, in a kind of super compound.

These distinctions suggest a qualitative difference between each type of work group and imply a change resulting from either or both internal and external conditions. My own lengthy experience in working with 
pluridisciplinary teams suggests that the liminal phase marking the transitions between forms of teaming is not the same as the routine kinds of dysfunction that plague many groups and teams. Liminal phases may be characterized by increased communication, heightened stress levels, misunderstanding, conflict and, possibly, renegotiation of the team's goals and objectives. Multidisciplinarity, interdisciplinarity, and transdisciplinarity as qualitatively different states exhibit distinct differences in levels of collaboration and engagement, and in the nature of the missions, goals, and objectives that are established.

The qualitative difference between one type of work group and another involves a shift in values and attitudes that allows for a reframing of the problem space, a willingness to accept diverse epistemologies, and an openness to considering multiple solutions. Each transition requires a change in the level of investment by each member. Multidisciplinarity requires the least investment: each member brings his/her individual knowledge base; negotiation is primarily in the area of operations and there is little expectation that members will invest time and energy in changing their perspectives. Interdisciplinarity requires a more significant investment of time and energy due to the need for more extensive negotiation in reaching shared understandings, which enables analysis and synthesis to occur across disciplinary boundaries. In the case of transdisciplinarity, the requirement for negotiation is very high: all members must be willing to subordinate their individual disciplinary perspectives to achieve a common vision that encompasses the dimensions and dynamics of an entire system. Figure 1 illustrates these differences.

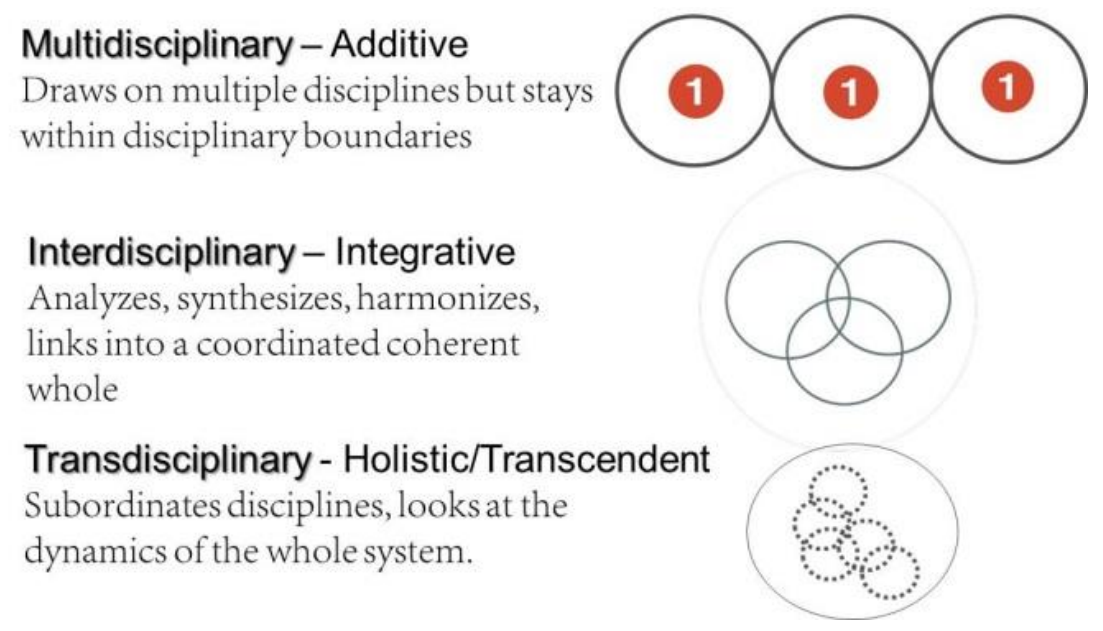

Figure 1: Types of pluridisciplinary teams: Additive, integrative, and holistic (Choi and Pak 2006) Visualization created by the author. 
As previously noted, Choi and Pak acknowledged that not every challenge requires a pluridisciplinary approach. However, they suggest several situations where pluridisciplinarity is desirable:

Situations in which it is necessary to resolve 'real world' or complex problems, when developing consensus in terms of definitions or guidelines is required, when there is a need to create a comprehensive prospective theorybased hypothesis for research, or when it is necessary to provide comprehensive services such as in health care or education (2006:357-358).

Climate change is a classic example of a complex problem, or what Rittel and Webber (1973) referred to as a "wicked problem," due to how it encompasses global and local environments that interact with each other in a myriad of ways. Such problems are ill-defined, uniquely configured, and involve multiple constituencies and interdependencies. Because of their multidimensional nature, the problems engendered by climate change cannot be solved by a single disciplinary approach-for example, by technologists-but must also incorporate social, cultural, environmental, economic, legal and political dimensions.

\section{The promise and problems of pluridisciplinarity}

The value of teams involving participants from diverse disciplines working together to solve complex problems is widely recognized (Marquardt and Horvath 2001, Benkler 2006, Choi and Pak 2006, Gloor 2006, Sawyer 2007, Miller, Aqeel-Alzrooni, and Campbell 2009, Sigelman 2009, Brown, Harris, and Russell 2010). However, less attention has been devoted to the work that precedes addressing the stated problem or team's objective, that is, the work required to integrate pluridisciplinary perspectives and enable collaboration. The social processes involved in reaching a state where participants not only cooperate, but also collaborate, can be described as "invisible work" (Forsythe 1999). Forsythe conceptualizes invisible work as the aspects that tend to be overlooked or invisible to those not trained in ethnographic research, which includes "detecting tacit knowledge" and assumptions (1999:130). There is often a tacit expectation that members of pluridisciplinary teams will work out whatever issues impede progress toward a solution to the stated problem. Sometimes the prior experience of one or more individuals is adequate to achieve the transition from an assemblage of participants to a working team. More often the obstacles to achieve highly integrated pluridisciplinary teams are insurmountable, resulting in achievements that are less than optimal and in the worst case, failure to achieve even moderate success (Contractor 2013).

These problems appear to be endemic for pluridisciplinary, multicultural teams. Marquardt and Horvath (2001:19) noted that: 
On most global teams, frustrations outnumber successes. Global executives, all too frequently, recount stories of setbacks caused by global teams, strategic plans that have suffered, careers that got derailed, projects that have taken so long that the competition took the market.

Tacit expectations that team members will "just work things out" overlook the root causes of the difficulties that participants are likely to encounter. Failure to recognize the formidable disciplinary and cultural differences that manifest in epistemologies, tools, languages, and work practices puts the teams and their projects at risk. Without the benefit of a seasoned guide, the prospect of arriving at a shared understanding of the mission and objectives, norms of communication, and agreements about how work will be done greatly diminish the chances of achieving an optimal outcome. Conklin (2006:15) refers to shared understanding about the problem and shared commitment around possible solutions as the "Holy Grail" of collaboration:

Shared understanding does not necessarily mean that we agree on the problem, although that is a good thing when it happens. Shared understanding means that the stakeholders understand each other's position well enough to have an intelligent dialog about the different interpretations of the problem, and to exercise collective intelligence about how to solve it.

\section{Pluridisciplinary teams: The ritual of collective work}

Liminality, described as a phase in the ritual process that is characterized as a state of "betwixt and between" (Turner 1995:95), provides a lens through which we might explore the stages of pluridisciplinary teamwork. The scope of this article limits the discussion of ritual to broad generalizations, specifically, that ritual is a fundamental component of social life in all societies, that it is exhibited in various types of rites, and that it is characterized by elements of performance, tradition, symbolism, prescribed rules, and formalization (Turner 1969, Geertz 1973, Douglas 1996, 2002). The concept of ritual has also been explored in the context of organizational culture (Gluesing 1995, Martin and Frost 1996, Cefkin 2010, Erwin 2015). The process of ritualization-that is, how activities and practices become ritualized-has been discussed indirectly and less frequently. Bell (1992:74) suggests that "ritualization is a way of acting that is designed and orchestrated to distinguish and privilege what is being done in comparison to other, usually more quotidian, activities." Bell's definition provides a useful distinction for comparing "regular" work groups and multidisciplinary collaborative teams. The ritualization of collective work, specifically in groups comprised of members from diverse disciplinary backgrounds, initially occurs in the act of bringing together a heterogeneous group of individuals for the purpose of 
engaging in a joint enterprise. Membership can be either voluntary or assigned. In an academic setting this might be a group of faculty from diverse disciplines; in an organization, it could be a cross-functional team. In either case, members of these groups are selected and in some fashion removed from their routine environments to become participants in a venture that requires the focused attention and input of diverse perspectives, training, and skillsets.

Wenger (1998:183) proposed that the role of ritual can be "understood in terms of community formation" and that

Rituals connect local practices and identities to other locations across time and space. They are a form of engagement that can bolster imagination-by cultivating the sense of others doing or having done the same thing-and alignment-by channeling an investment of the self into standardized activities, discourses, and styles.

Wenger's concept, tying ritual and community formation together, anticipates the social processes involved in preparing for collaboration. How a group self-organizes, how it negotiates the rules that will govern members' interactions, the ways in which activities are ordered, and the meanings that are ascribed to particular language, symbols, and artifacts, exemplify the process of ritualization of collective work practice.

Extending the concept of ritual to collective work implies that processes and practices be "formalized" in some way, Meerwarth et al. (2005) discuss the difference between formal codified rules and the "unwritten partnership rules" that emerge during partnership formation. They note that "emergent partnership rules are individually-based, independently-generated, voluntary perceptions and assessments of partnership" (p. 288). Since they are not formalized, they can be accepted or rejected and are enforceable only through the consensus of the group. Because they are unwritten, partnership members might not even be aware of them.

If we think of the process of collective work done by individuals from multiple disciplines as a form of ritualized practice, then the space created by the qualitative shifts that occur in transitioning from one phase of team interaction to another might be construed as liminal space. The team is neither what it was, nor what it might become-an active state rather than one in which activity is suspended. What occurs in the process of transitioning is a "black box" of social interaction in which team members must engage in three critical tasks (Miller 2012) to perform beyond their singular disciplinary perspectives:

1. Articulate the value of their disciplinary perspective in relation to the goals and objectives of the project;

2. Demonstrate the use and value of the disciplinary practices and tools they contribute; and 
3. Engage in negotiating, integrating, and managing diversity in environments where the team's multiple knowledge domains are in play.

Accomplishing these tasks requires that participants be willing to engage in an intense social learning process.

In pluridisciplinary collective work the process of formalizing rules occurs as a result of participation and through the reification of the operational aspects of collective work and the products that are generated by the joint enterprise (Wenger 1998). If organization occurs, structure and formalization emerge from what is initially organic and informal (Stinchcombe 2001).

The liminal phase as threshold

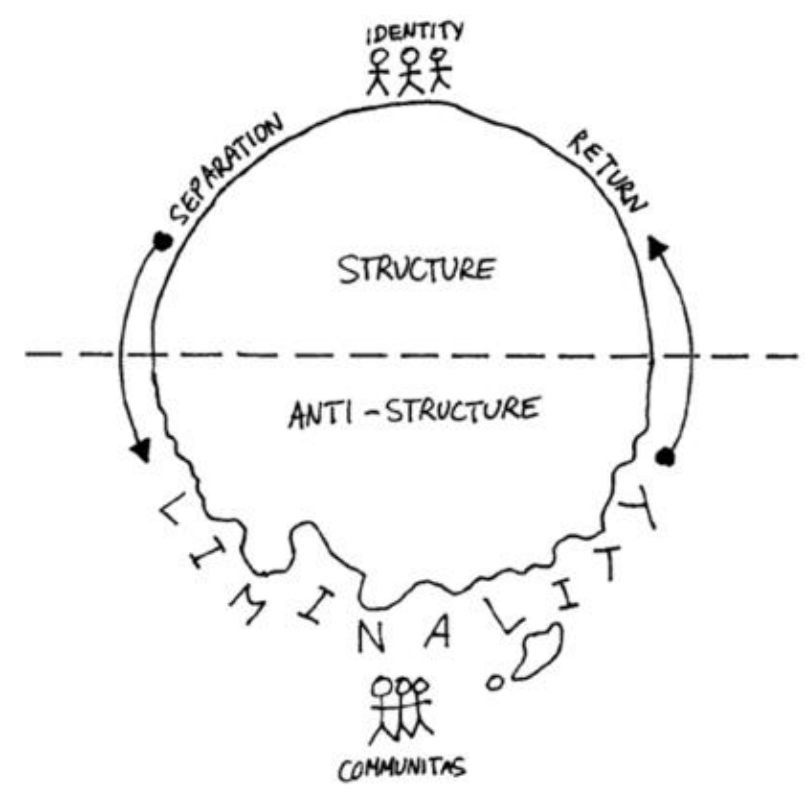

Figure 2: The Rite of Passage

(Source: Illustration provided by wearenotconnected.wordpress.com)

Conceiving of the transitory stages in the process of collective work through the lens of liminality provides a framework in which to study why and how such transitions occur. The term liminality is derived from the Latin limen meaning "threshold." The liminal state is described as a phase during a rite of passage in which individuals engaged in a rite of passage move from the separation phase and enter the margin (or threshold) (Turner 1995). In the first of three phases (separation, margin, and aggregation) identified by van Gennep, individuals or "initiates" are removed from the structure and norms of their customary 
lives and identities. Once separated from the normal routines and structure of their lives, they enter a realm in which they may experience certain elements of "anti-structure" (Turner 1995:96) including role reversal, confusion, humiliation, and, sometimes, abuse. Removed from former social roles and norms, participants are bound to each other through what Turner (1995:96) refers to as "communitas," a shared experience in which they all partake equally. As depicted in Figure 2, the rite of passage concludes with the re-entry of the initiates into the structure and norms of the community, but with a new identity with commensurate changes to their social position, responsibilities, rights and privileges.

\section{Innovation 101: Heterogeneity versus homogeneity}

A growing awareness of the limitations of singular forms of inquiry to address the complex problems that societies and organizations are currently facing (Wilson and Pirie 2000, Marquardt and Horvath 2001, Davies 2006) is fueling the interest in pluridisciplinary teams.

Comprehension of the scope and depth of these problems requires a holistic or systems perspective, one that allows problems to be viewed simultaneously from multiple diverse perspectives. This might be conceptualized by applying the metaphor of a diamond: viewing the "stone" through its various facets reveals different aspects of the problem. Brown et al. (2010) posit that "The task is...to draw on all our intellectual resources, valuing the contribution of all the academic disciplines as well as other ways in which we construct our knowledge."

In discussing the nature of communication flows through interpersonal networks, Rogers introduces the concepts of "homophily" and "heterophily." Rogers notes that the labels were first introduced by Tarde (1903) who argued that "Social relations I repeat, are much closer between individuals who resemble each other in occupation and education." Rogers (2003:305) defines homophily as "the degree to which a pair of individuals who communicate is similar." Weighing the benefits of homophily and heterophily, he notes that "heterophilous networks, those that are composed of individuals who are different in certain attributes, have special informational potential, even though it may occur only rarely" (Rogers 2003:306). While heterophilous groups are likely to experience more difficulty in communication due to cognitive dissonance resulting from differences in technical competence, language, and tools, these networks have the greater potential when it comes to diffusing innovation. Rogers concludes that the "bridges" or interpersonal links that they create in a communication system are able to span multiple networks to convey information about innovations, as Granovetter's (1973) theory of the strength of weak ties suggests.

The growing awareness that multiple, rather than singular, viewpoints are best suited to address "wicked problems" has engendered 
the practice of deploying teams comprised of participants from multiple disciplines. Wicked problems constitute a unique class of problems that require an approach that transcends traditional disciplinary perspectives, but this is easier said than done. Unfortunately, we assume more than we know: ignoring the work required to integrate pluridisciplinary perspectives in a way that allows teams to creatively overcome compartmentalized epistemologies and singular modes of inquiry puts teams at risk of not only achieving marginal success, but also of failing completely.

New realities, new requirements

According to Castells (2000), $21^{\text {st }}$ century organizations face challenges that are unique to this era-including discontinuous rather than incremental change, emergent over prescribed order, simultaneous attention to both local and global concerns, and the growing dominance of networks over bounded silos. Advances in information and communication technologies are accelerating the rate of change that impacts competitive market and global conditions and dictates new organizational capabilities. These include team-based collective work, cultural competence, new forms of leadership, accountability, a high tolerance for ambiguity and uncertainty, sensitivity to social processes, continuous learning, and the ability to communicate "The New" in multiple modes, including verbal, visual, text and multimedia formats (Erwin 2014). Organizational norms, processes, structures and values can be designed either to support or inhibit these practices and competencies. Relating this to pluridisciplinary group work: an openness to recognizing and accepting the validity of different knowledge cultures is essential for these teams to be productive.

Many of the required skills and competences are considered "soft skills"-such as humor, empathy, emotional intelligence and optimism-which are assumed to be personal attributes that are difficult to test or assess in a job candidate. Although more organizations are recognizing their importance (Caudron 1999), soft skills continue to be nice-to-have rather than got-to-have qualities in screening individuals for employment. However, there is a strong relationship between so called soft skills and the characteristics common to effective members of pluridisciplinary teams because they tend to be indicators of aptitudes for leadership, teamwork, negotiation, communication, and sociability. These kinds of skills are useful when navigating the rough waters caused by "shared misunderstandings" (Batteau 1979) arising from disciplinary differences related to language, epistemology, meaning, tools, and work practices. Organizations that provide opportunities to learn and practice soft skills may be better positioned to foster organizational cultures that value and reward people who develop the interpersonal skills. Such an 
organizational culture can aid in the transitioning process through the stages of multidisciplinary teamwork.

\section{In practice the work is messy}

Working late one night, Conklin encountered a janitor who commented that the vacuum cleaner he was using was not working as it should: it was not actually picking up the dirt. Later Conklin (2006:3) made the analogy that "When we are working on wicked problems in a socially complex environment [e.g., pluridisciplinary teams], it is much harder to notice that our tools are simply not 'picking up the dirt.'” The advantage that the janitor had was that he could clearly see the dirt the vacuum was missing.

Gluesing (1995) described the relationships between members of pluridisciplinary cross-functional teams as "fragile alliances." Her study of a global virtual team (GVT) in a multinational high tech firm explored how GVT members made sense of the cultural complexity of their work and how they operated within that complexity. In addition to coming from diverse disciplinary backgrounds, these teams were separated by physical and psychological distance, corporate boundaries, and cultural differences. Gluesing's study suggests that preparing team members to deal with these conditions and factors prior to their experience might have resulted in a positive outcome.

\section{Collaborative Innovation Networks (COINs) Seminar: A multi-year study of pluridisciplinarity}

The COINs graduate seminar ${ }^{1}$ provided an opportunity to study pluridisciplinary teams in a global virtual partnership. The seminar is taught annually in partnership with MIT Sloan School of Management and the Center for Collective Intelligence, the University of Cologne, the University of Bamberg, the Stuart School of Business as the Illinois Institute of Technology, and Aalto University in Helsinki. The seminar has been conducted annually since 2005 and has sometimes involved other academic partners and clients. Personal experience from 2008 through 2014 as a faculty partner in the Collaborative Innovation Networks (COINs) graduate seminar has led to insights that indicate a need for explicit learning opportunities that prepare participants to collaborate effectively in pluridisciplinary teams. Guiding teams through the challenges inherent in multicultural, pluridisciplinary teaming made clear the need to train participants in the skillsets required to reach agreement on mission, goals, and objectives, to negotiate shared meaning, and to build the operational structure needed for the team to function. For example, in the 2013 COINs seminar, an American female design management student was teamed with a group of four male German

\footnotetext{
${ }^{1}$ https://sites.google.com/site/coincourse2015/home
} 
engineering students. Despite repeated attempts by the female student to contribute to the project, her ideas were given cursory consideration and rarely if ever implemented. The German teammates continued to use German as their primary language even when the female student was involved. The female student declined an intervention by the faculty coaches. Rather than participating fully, she opted instead to provide whatever contributions she could and left important decisions about implementation to her German counterparts.

Insights gained through years of participating in the partnership suggest that the seminar itself created a context that allowed for engaging in both theory and practice. Four years after the partnership began, I concluded in a narrative case study that:

Significant opportunities for learning are rooted in the multidisciplinary and multicultural context of the seminar and the interactions that occur between GVT [global virtual team] members. Lessons learned underscore the importance of social learning as a means of identifying and understanding the mechanisms that facilitate or inhibit collaboration at the team level. Insights from the seminar have led us to think about how the linkage between social learning, collaboration, and the knowledge sharing challenges encountered by multidisciplinary global virtual teams might be integrated to form a theoretical framework to guide the development of explicit learning objectives (Miller 2012:132).

How might we make sense of the "messiness" that most multidisciplinary teams experience? One possible way is to equate this "messiness" to the notion of "anti-structure," a characteristic of the liminal state in traditional rites of passage during which initiates experience role reversal, ego deflation, and other forms of disorientation. Referring again to the illustration in Figure 2, we might imagine team members leaving work groups in which their roles and identities are established to join a new group whose members are socialized and educated in disciplines different from their own. For example, graduate students participating in the COINs seminar come from design, business, programming, and computer science departments. The newly formed teams take on a project. However, before the "real work" can begin, team members must sort out what exactly what their mission is, what objectives will need to be met, and how they will organize to achieve success. As can be seen in Figure 2, the journey begins through liminal territory where confusion and conflict (anti-structure) reign. No longer among others who share the same language, tools, and work practices, team members face the tasks of articulating, demonstrating, negotiating, and integrating. During the process of reaching consensus the team sets itself on a path to reach a level of engagement and collaboration, either multidisciplinary (additive), 
interdisciplinary (integrative), or transdisciplinary (transcendent or holistic).

We might think of the initial phase of the work of social learning that pluridisciplinary teams confront as being "the mess," described by Erwin (2014) as what is deemed "the problem," but is not yet understood or clearly articulated. The mess is unfamiliar, "still fuzzy," unpredictable, and complex. It is a liminal space through which team members must pass to transition from one phase to another, depending on the level of engagement and collaboration that they collectively aspire to reach. According to Erwin (2014:2), "the mess" is what precedes "The New": that which is recognized as vitally important, has stature, and receives respect and attention. Erwin notes: "there is a lot less glory and guidance in the mess." The mess is familiar to most pluridisciplinary teams. It is unlike the rituals we are familiar with from studies of traditional societies because in contemporary rituals, like pluridisciplinary teaming, guidance is relatively rare. Glory is even rarer.

\section{Managing complexity and turbulence in pluridisciplinary teams}

A growing number of firms have accepted that pluridisciplinary teams provide an alternative means of tackling the complex problems and challenges facing organizations today. Practitioners and scholars have called for new forms of leadership that allow organizations to adapt to rapidly changing conditions (Southern, Gaffney, and Moore 2012). In pluridisciplinary teams, for example, this might take the form of rotating leadership among team members based on the particular skillsets that are required at certain times during the project. In addition to internal leadership, there is growing recognition of the importance of external guidance and mentoring for, as Marquardt and Horvath (2001:157-158) point out:

Often we point to the team leader for guidance. Team leaders, however, have their hands full with managing and administering the activities and tasks of the group. More important, a leader is usually right in the middle of the action-a position that doesn't usually offer a holistic perspective. It's difficult to understand conflict or communication issues when you're part of the process (and indeed may be part of the problem).

Imagining the skills and qualities of the individuals who serve as ritual guides and facilitators in traditional societies can help to identify the requirements for guiding and mentoring pluridisciplinary teams. Unfortunately, job descriptions for ritual guides are rare. What we do know is that a ritual guide would be an individual who has been through the ritual process and, consequently, is sensitive to what an initiate is likely to experience. If we consider multidisciplinary collective work as a form of ritual, we might assume that guiding and mentoring these teams 
requires similar qualifications, including the ability to observe and interpret social processes and interact with team members in ways that help them to build consensus, resolve conflicts and achieve clarity when goals and objectives become clouded. The ideal guide or mentor is able to serve as a resource and sounding board for the team and facilitate group processes when required. To serve as an unbiased observer the guide also must be aware of his or her own personal assumptions. Finally, the guide must be able to achieve analytical distance: to stand apart without standing above (in the hierarchical sense).

Such individuals do exist and their numbers are growing. There is also an increasing number of examples of teams that have successfully negotiated the transitions from multidisciplinary to interdisciplinary and transdisciplinary, as illustrated in Figure 1. A number of firms-particularly design consultancies like IDEO, Gravity Tank, Conifer, and Iota-have formed with the intention to deploy pluridisciplinary research teams. The Research Network for Design Anthropology ${ }^{2}$ centered in Denmark was founded on a commitment to exploring pluridisciplinary teaming practices, specifically between designers and anthropologists, but also incorporating a diverse range of disciplinary perspectives in project-based work. Some organizations have been deploying pluridisciplinary teams for many years and the success rate for these teams has improved dramatically over time as more of their employees and members have become seasoned and experienced, and as organizational environments and structures have adapted to the requirements and practice of pluridisciplinarity.

Examples of successful pluridisciplinary/multifunctional teams are featured in a forthcoming volume on collaboration. It includes a collection of works that "address roles that business anthropologists assume as choreographers or participants in collaborative ethnography when they work in and with corporations and other organizations" (McCabe 2016). From a variety of perspectives and contexts, the articles describe situations that illustrate how working with pluridisciplinary and multifunctional teams "is a dynamic process of social interaction" involving knowledge, power and emotion. In the Introduction, McCabe explains how anthropologists live in "liminal space" in situations that require "cognitive convergence," requiring that they read and translate other perspectives, "shifting back and forth between participation and observation."

McCabe introduces the concept of "cognitive convergence", which is a state of shared understanding that must be reached for team members to communicate effectively across disciplinary and functional boundaries. The purpose of this article is to explore the process by which cognitive convergence is achieved. Applying liminality as a framework to

2 https://kadk.dk/co-design/research-network-design-anthropology 
conceptualize a state of "betweenness" opens a space within which we might explore how shared understanding and "shared misunderstandings" (Batteau 1979) are processed by individuals and the team.

The emergence of practitioner and academic organizations, training programs, and movements provide evidence of the increasing interest in pluridisciplinarity. One of the best known academic organizations is the Science of Team Science (SciTS), described as "a rapidly growing cross-disciplinary field of study that aims to build an evidence-base and to develop translational applications to help maximize the efficiency and effectiveness of team based research." ${ }^{3}$ The Balanced Team movement is described as a "self-organizing group" and "a global movement of people who value multidisciplinary collaboration and iterative delivery focused on customer value as a source for innovation." 4 Its primary areas of interest include Lean Startup, Agile, Lean, Agile UX, Lean UX, Devops (development operations), and Customer Development.

\section{The challenge for applied anthropologists}

Pluridisciplinarity is emerging as a field for which anthropologists are particularly suited. Describing the challenges of collaboration within multinational firms to meet customer needs, Peinado (2014) argues that "Given their understanding of complex cultural systems, anthropologists have much to contribute to how organisations can transform themselves in order to adapt to these new challenges." The holistic perspective that is fundamental to anthropologists' training equips them to identify, process, and communicate the complexity inherent in multidisciplinary and multifunctional teams. Being both an active participant as well as an observer can result in key insights regarding group processes that either facilitate or inhibit progress through the interdisciplinary, multidisciplinary, and transdisciplinary stages of team development.

As the demand for applied anthropology has increased, anthropologists have moved into ever more active roles. This shift has moved anthropologists from external observers and traditional participant observation into the domain of active engagement as organizational members. Even more active participation-such as in the emerging field of design anthropology-moves anthropologists from observers to interventionists engaging in transformative practices (Gunn, Otto, and Smith 2013). Many anthropologists have served as members of pluridisciplinary teams. The guide might be a peer or core group member, or may be external in the manner of a traditional ritual guide-a member of the community that has been through the ritual experience and is able to assist others in navigating the process. The goal is to reach a shared

3 http://www.scienceofteamscience.org/, accessed on August 20, 2015.

${ }^{4}$ http://www.balancedteam.org/, accessed on August 20, 2015. 
understanding of the reasoning and rationale behind divergent ideas and practices. As McCabe (2016: in press) explains "such roles are challenging because they involve the intersection of different sources of knowledge, power and emotion. Since knowledge, power and emotion are social constructions, they require reading and translation when people work together."

An anthropologist, or a researcher from any discipline, who has experience in successfully negotiating the process of pluridisciplinary collaboration, can serve in the role of guide. After all, ritual guides in traditional societies were not anthropologists. The question is how can the skills, sensitivities, and theoretical perspectives and frameworks particular to educating and training anthropologists-and others-be applied to guide groups through the stages of multidisciplinary, interdisciplinary, and transdisciplinary teamwork as illustrated in Figure 1.

Nevertheless, anthropologists are uniquely qualified to serve as guides in helping teams navigate the liminal states that mark the transitions between multidisciplinary, interdisciplinary, and transdisciplinary teaming. They are experienced as observers of the dynamic and fluid states of social interaction and sensitive to cultural nuances. Much anthropological research is conducted with the aim of describing and interpreting "the other"; traditionally, this has meant describing non-Western societies to the West. With the emergence of subdisciplines, such as business and design anthropology, the anthropological gaze has turned increasingly to contemporary societies, cultures, and subcultures. For example, in describing the role of culture in the process of innovation and adaptation in the automotive industry, Briody et al. (2010) specify the importance of anthropological fieldwork in examining cultural change and transformation.

Serving in the role of ritual guide, anthropologists can assist team members to process and make sense of their experiences and provide help in articulating the nature of engagement, establishing shared routines and practices, dealing with conflicts, developing strategy, negotiating shared meaning and models, and navigating the transitions between multidisciplinarity, interdisciplinarity, and transdisciplinarity. With access to the vast archive of anthropological knowledge-ethnographies, theories, methodologies, and concepts-developed through intimate in situ engagement with social groups across time and space, anthropologists can draw on a wealth of resources. Over the course of their studies, student anthropologists are exposed to these archives, which constitute the rich legacy and history of the field.

Acting as ritual guides requires some reimagining of anthropology. However, this shift is already well under way as anthropologists move from roles as observer-participants to 
incorporating intervention and transformative practices in collaboration with other disciplines (Kilbourn 2013). There is general agreement that pluridisciplinary teams composed of diverse disciplinary members can be effective in tackling so called "wicked" problems (Conklin 2005). Bringing individuals from diverse disciplines together in pluridisciplinary teams creates the potential for innovation (Rogers 2003, Strathern 2004). While this trend is increasingly important, it is also problematic. As Gunn and Clausen (2013) argue, "we question the idea that if you just bring different knowledge traditions together this will lead to innovation-it is not that easy." The challenges confronting pluridisciplinary teams continue to hinder team members from successfully navigating the transitions from multidisciplinary to interdisciplinary, and to achieving the final leap to transdisciplinary teaming.

\section{Conclusion}

The point has been made that not all situations require the attention of a multidisciplinary team. Although the progression through multidisciplinarity, interdisciplinarity, and transdisciplinarity as illustrated in Figure 1 is neither guaranteed nor compulsory, we are nonetheless left with the sense that the type and scale of the problems that human societies are confronting require a holistic approach that transcends disciplinary biases, assumptions, and barriers. Ever more pressing systems-level problems of the social and environmental variety have immense implications for business organizations and society at large. The need to generate and apply actionable knowledge that transcends disciplinary boundaries-to evolve beyond the current model of singular forms of inquiry-has become an imperative. There is no set formula or algorithm for achieving this goal. Everything depends on the particular context and experience of the individuals who happen to be involved.

However, there are many examples of how this imperative is being addressed through formal organizations such as the SciTS, and practitioner-focused groups like the Balanced Team movement. Approaches such as transdisciplinary team-based research, which aims "to integrate and ultimately extend beyond discipline-specific concepts, approaches, and methods to accelerate innovations and progress toward solving complex real-world problems," are being embraced in medical and other research fields (Hall et al. 2012). Organizations can access resources and guidance from these and other sources.

A simple checklist provides initial practical direction for organizations that intend to utilize pluridisciplinary teams or to improve the effectiveness of existing cross-disciplinary teamwork. First, assess the nature of the projects under consideration: do they require the involvement of pluridisciplinary perspectives and skill sets? Analyze the dimensions-for example, social, technological or cultural-and the 
research areas that are involved. Next, discuss who might be qualified for the team. Of those potential participants, who has previous experience with the research topic? Who has either prior experience working in pluridisciplinary collaborations or a willingness to engage in such work? Identify the person who will take the role of facilitator/guide or coach for the team. Will it be a member of the team or someone external? A prelaunch meeting of potential teammates with the facilitator/guide to discuss the project and the contributions that each would bring is essential. The return on the initial investment of time in following through on these suggested steps is borne out in literature on the outcomes of successful pluridisciplinary teams. Much depends on the compatibility of the individuals, which remains an unknown until people actually come together. Even though a candidate might have expertise in a particular research area, if he or she is not willing or able to engage with others in a way that allows each member's contribution to be respected and considered, that candidate is not likely to be a productive addition to the team.

We can apply the phases involved in rites of passage (Turner 1995) to understand teamwork effectiveness. Confirming the selection of team members can be considered the beginning of the separation or detachment phase. Individuals enter the liminal phase once the project is underway. This state is characterized by blurring or confusion of disciplinary distinctions. Turner (1995:94) notes that during the liminal period 'the characteristics of the ritual subject (the 'passenger') are ambiguous; he passes through a cultural realm that has few or none of the attributes of the past or coming state." The third phase-re-aggregation or reincorporation-completes the process: passage is consummated.

A pluridisciplinary team, like any team, will encounter problems in both teamwork-the social interactions among team members; and task work-the set of tasks that must be accomplished to reach a desirable output. The role of the facilitator/guide or coach is to assist team members in negotiating the teaming process, in resolving conflicts and overcoming barriers to achieve a cohesive final product that represents the best thinking and skills of the individual participants. How the participants are able to achieve the levels of teaming depends upon: (1) the nature of the project; (2) the level of skills and the commitment of individual team members to an integrated outcome; and (3) the ability of the facilitator/guide to help the team make the transitions from multidisciplinary to interdisciplinary, and from interdisciplinary to transdisciplinary.

We might look to the past for inspiration and guidance. If we imagine collective work as a form of ritual, we might study the role of the ritual guides who shepherded initiates through the confusion and conflicts inherent in navigating transitional stages and liminal spaces. By balancing the need to break established identities, structures, and 
routines, that is, to create anti-structure, we allow for the emergence of new forms of working, new types of identity, and new perspectives on the world. Can we learn or imagine how they were able to achieve balance at the edge of chaos? It might then be possible to design interventions through mentoring and guiding that would improve the odds for pluridisciplinary teams to evolve beyond multidisciplinary to reach, when appropriate, interdisciplinary and transdisciplinary states.

\section{References}

Barfield, Thomas, ed. 1977. The Dictionary of Anthropology. Oxford: Blackwell Publishers Ltd.

Batteau, Allen. 1979. "Appalachia and the Concept of Culture: A Theory of Shared Misunderstandings." Appalachian Journal 7 (1/2):9-31. doi: $10.2307 / 40932719$.

Bell, Catherine. 1992. Ritual Theory, Ritual Practice. Oxford: Oxford University Press.

Benkler, Yochai. 2006. The Wealth of Networks: How Social Production Transforms Markets and Freedom. New Haven: Yale University Press.

Briody, Elizabeth K., Robert T. Trotter, and Tracy L. Meerwarth. 2010. Transforming Culture: Creating and Sustaing Effective Organizations. New York: Palgrave Macmillan.

Brown, Valerie A., John A. Harris, and Jacqueline Y. Russell, eds. 2010. Tackling Wicked Problems Through the Transdisciplinary Imagination. New York: Earthscan.

Caudron, Sherri. 1999. "The Hard Case for Soft Skills." Workforce 78 (7):60-66.

Cefkin, Melissa, ed. 2010. Ethnography and the Corporate Encounter: Reflections on Research in and of Corporations. Edited by Sarah Pink and Simone Abram. 5 vols. Vol. 5, Studies in Public and Applied Anthropology. New York: Berghahn Books.

Choi, Bernard C.K., and Anita W.P. Pak. 2006. "Multidisciplinarity, interdisciplinarity and transdisciplinarity in health research, services, education and policy: 1 . Definitions, objectives, and evidence of effectiveness." Clinical \& Investigative Medicine 29 (6):351-364.

Conklin, Jeff. 2005. Wicked Problems and Social Complexity. In from Dialogue Mapping: Building Shared Understanding of Wicked Problems: Wiley.

Conklin, Jeff. 2006. Dialogue Mapping: Building Shared Understanding of Wicked Problems. New York: John Wiley \& Sons. 
Contractor, Noshir. 2013. "Some Assembly Required: leveraging Web science to understand and enable team assembly " Philosophical Transactions of the Royal Society A 371 (1987):1471-2962

Davies, E. Brian. 2006. Epistemological Pluralism. London: PhilSci Archive.

Douglas, Mary. 1996. Natural Symbols. New York: Routledge.

Douglas, Mary. 2002. Purity and Danger: An analysis of concept of pollution and taboo. New York: Routledge.

Erwin, Kim. 2014. Commuicating the New: Methods to Shape and Accelerate Innovation. Hoboken, NJ: John Wiley \& Sons.

Erwin, Kim. 2015. Kim Erwin Interview. edited by Chris Miller.

Forsythe, Diana E. 1999. "'It's Just a Matter of Common Sense": Ethnography as Invisible Work." Computer Supported Cooperative Work 8:127-145.

Geertz, Clifford. 1973. The Interpretation of Cultures. New York: Basic Books.

Gibbons, Michael, Camille Limoges, Helga Nowotny, Simon Schwartzman, Peter Scott, and Martin Trow. 1994. The New Production of Knowledge: The Dynamics of Science and Research in Contemporary Sociteties. London: Sage.

Gloor, Peter. 2006. Swarm Creativity: Competitive Advantage through Collaboration Innovation Networks. New York: Oxford University Press.

Gluesing, Julia. 1995. "Fragile alliances: negotiating global teaming in a turbulent environment " Ph.D. Doctoral, Anthropology, Wayne State University.

Granovetter, Mark S. 1973. "The Strength of Weak Ties." Americn Journal of Sociology 78:1360-1380.

Gunn, Wendy, and Christian Clausen. 2013. "Conceptions of Innovation and Practice." In Design Anthropology: Theory and Methods, edited by Wendy Gunn, Ton Otto and Rachel C. Smith, 159-179. New York, NY: Bloomsbury.

Gunn, Wendy, Ton Otto, and Rachel Charlotte Smith, eds. 2013. Design Anthropology: Theory and Practice. New York: Bloomsbury

Hall, KaraL, AmandaL Vogel, BrookeA Stipelman, Daniel Stokols, Glen Morgan, and Sarah Gehlert. 2012. "A four-phase model of transdisciplinary team-based research: goals, team processes, and strategies." Translational Behavioral Medicine 2 (4):415-430. doi: 10.1007/s13142-012-0167-y.

Johnson, Neil F. 2009. Simply Complexity: A Clear Guide to Complexity Theory. Oxford: Oneworld Book. 
Kilbourn, Kyle. 2013. "Tools and Movements of Engagement: Design Anthropology's Style of Knowing." In Design Anthropology: Theory and Practice, edited by Wendy Gunn, Ton Otto and Rachel C. Smith, 68-82. New York, NY: Bloomsbury.

Lawrence, Roderick J. 2010. "Beyond Disciplinarity Confinement to Imaginative Transdisciplinarity." In Tackling Wicked Problems Through the Transdisciplinary Imagination, edited by Valerie A. Brown, John A. Harris and Jacqueline Y. Russell, 16-30. New York: Earthscan.

Margolin, Victor. 2002. The Politics of the Artificial: Essays on Design and Design Studies. Chicago: University of Chicago.

Marquardt, Michael J., and Lisa Horvath. 2001. Global Teams: How top multinationals span boundaries and cultures with high-speed teamwork. Palo Alto: Davies-Black Publishing.

Martin, Joanne, and Peter Frost. 1996. "The Organizational Culture War Games: A Struggle for Intellectual Dominance." In Studying Organization: Theory \& Method, edited by Stewart R. Clegg and Cynthia Hardy. Thousand Oaks: Sage Publications.

McCabe, Maryann. 2016. "Collaborative Ethnography: Intersection of Knowledge, Power and Emotion." In Collaborative Ethnography in the Business Environment, edited by Maryanne McCabe, in press. Walnut Creek, CA: Left Coast Press.

McGregor, Sue L.T. 2014. The Transdisciplinary Meme. Integral Leadership Review 16 (3). Accessed April- June 2014.

Meerwarth, Tracy L., Elizabeth K. Briody, and Devadatta M. Kulkarni. 2005. "Discovering the Rules: Folk Knowledge for Improving GM Partnerships." Human Organization 63 (3):286-302.

Miller, Christine. 2012. "Lessons learned and future directions: designing for multidisiplinarity in collaborative innovation networks (COINs)." International Journal of Organizational Design and Engineering 2 (2):132148.

Miller, Christine, Saad Aqeel-Alzrooni, and R.W. Campbell. 2009.

"Learning to Collaborate in COINs: Insights from a multidisciplinary global virtual collaboration." Collaboration Innovation Networks Conference, Savannah, GA.

Nowotny, Helga, Peter Scott, and Michael Gibbons. 2001. Re-thinking Science: Knowledge and the Public in an Age of Uncertainty. Cambridge: Polity.

Peinado, Alice D. 2014. "Collaborating Across and Beyond the Corporation via Design Anthropology." International Journal of Business Anthropology 5 (1):70-78.

Rogers, Everett M. 2003. Diffusion of Innovations. New York: Free Press. Reprint, 5th edition. 
Sawyer, Keith. 2007. Group Genius: The creative power of collaboration. New York: Basic Books.

Sigelman, Lee. 2009. "Are Two (or Three or Four ... or Nine) Heads Better than One? Collaboration, Multidisciplinarity, and Publishability." Political Science and Politics 42 (3):507-512.

Southern, Nancy, Sylvia Gaffney, and Bernice Moore. 2012. "Leaning into Complexity: Supporting Leaders Through Transformative Learning." Integral Leadership Review 11 (11):9.

Stinchcombe, Arthur L. 2001. When Formality Works: Authority and Abstraction in Law and Organizations. Chicago: University of Chicago Press.

Strathern, Marilyn. 2004. Commons and Borderlands: Working Papers on Interdisciplinarity, Accountability and the Flow of Knowledge: Sean Kingston Publishing.

Strathern, Marilyn. 2007. "Interdisciplinarity: some models from the human sciences." Interdisciplinary Science Review 32 (2):123-134.

Tarde, Gabriel. 1903. The Laws of Immitation. Translated by Elsie Clews Parson. Rahway, N.J.: The Mershon Company Press.

Tuckman, Bruce W., and Mary Ann C. Jensen. 1977. "Stages of Small-Group Development Revisited." Group \& Organization Studies (pre-1986) 2 (4):419-427.

Turner, Victor. 1969. The Ritual Process: Structure and Anti-Structure. Chicago: Aldine Publishing Company.

Wenger, Etienne. 1998. Communities of Practice: Learning, Meaning and Identity. Edited by John Seely Brown, Learning in Doing: Social, Cognitive and Computational Perspectives. Cambridge, UK: Cambridge University Press.

Wilson, Valerie, and Anne Pirie. 2000. Multidisciplinary teamworking indicators of good practice: Scottish Council for Research in Education.

Christine Z. Miller, Ph.D., is Clinical Associate Professor of Innovation at IIT Stuart School of Business. Previously, Chris served on the faculty in the graduate program in Design Management at the Savannah College of Art and Design (SCAD). Her interdisciplinary doctoral research (Anthropology and Management) explored process formalization and the relationship between innovation and formalization at a Tier One automotive supplier. Her research interests incorporate how sociality and culture influence the design and diffusion of new products, processes, and technologies. She studies technology-mediated communication and knowledge workflows within pluridisciplinary groups, teams, and networks and the emergence of collaborative innovation networks (COINs). Miller is a founding member of the COINs conference. She can be reached at cmille31@iit.edu. 